\title{
On the Growth of Dust Particles in a Non-Uniform Solar Nebula
}

\author{
Nader Haghighipour \\ Department of Terrestrial Magnetism and NASA Astrobiology Institute, \\ Carnegie Institution of Washington, 5241 Broad Branch Road, Washington, DC 20015
}

\begin{abstract}
A summary of the results of a numerical study of the growth of solid particles in the vicinity of an azimuthally symmetric density enhancement of a protostellar disk are presented. The effects of gas drag and pressure gradients on the rate of growth of dust particles and their settling on the midplane of the nebula are also discussed.
\end{abstract}

\section{INTRODUCTION}

It has recently been shown that the combined effect of gas drag and pressure gradients causes solid objects to rapidly migrate toward the location of maximum pressure in a gaseous nebula $[1,2]$. Such rapid migrations result in the accumulation of objects in the vicinity of local pressure enhancement and may increase the rates of their collisions and coagulations. In a disk with a mixture of gas and small solid particles, the motion of an object is also affected by its interaction with the particulate background. An object may, in such an environment, sweep up smaller particles and grow larger during its gas-drag induced migration. In this paper I present a summary of a study of the growth-rates of dust grains to centimeter-sized objects in a nebula with a local pressure enhancement.

\section{THE MODEL NEBULA}

An isothermal and turbulence-free protostellar disk is considered here with a solar-type star at its center. The nebula is assumed to be a mixture of pure molecular hydrogen at hydrostatic equilibrium, and small submicron-sized solid particles. The density of the gas in this nebula is considered to be

$$
\rho_{g}(r, z)=\rho_{0} \exp \left\{\frac{G M m_{H}}{K_{B} T}\left[\frac{1}{\left(r^{2}+z^{2}\right)^{1 / 2}}-\frac{1}{r}\right]-\beta\left(\frac{r}{r_{m}}-1\right)^{2}\right\} .
$$

In equation (1), $M$ is the mass of the central star, $G$ is the gravitational constant, and $K_{B}, T$ and $m_{H}$ represent the Boltzmann constant, the gas temperature, and the molecular mass of hydrogen, respectively. The quantities $\rho_{0}, r_{m}$ and $\beta$ in equation (1) are parameters of the system with constant values. The gas density function as given by equation (1) ensures that along the vertical axis, the gravitational attraction of the 
central star will be balanced by the vertical component of the pressure gradients [2]. It also has azimuthally symmetric gas-density-enhanced regions on any plane parallel to the midplane. On the midplane, the density of the gas maximizes at $r=r_{m}$.

The particles of the background material of a nebula are strongly coupled to the gas and their motions are only affected by the gas drag. For the submicron-sized particles considered here, the rate of the gas drag induced migration is so small that one can assume, at any position in the nebula, the gas and particle distribution functions are proportional. That is, $\rho_{\text {dust }}(r, z)=f \rho_{g}(r, z)$, where $\rho_{\text {dust }}(r, z)$ is the distribution function of the background material. The solid-gas ratio $f$ is taken to be constant and equal to 0.0034 .

\section{EQUATION OF MOTION}

For an object larger than the submicron particles of the background material, the abovementioned gas-particle coupling is less strong. As a result, the object moves faster than the background particles and may collide with them. Such collisions may result in adhesion of the background particles to the moving object and increase its mass.

The rate of change of the momentum of an object due to the sweeping up of the background material is proportional to the rate of the collision of the moving object with those particles. Assuming the sticking coefficient is equal to unity, one can write,

$$
\frac{d \mathbf{P}}{d t}=\pi \rho_{\text {dust }}(r, z) a^{2} \frac{d \ell}{d t}(\mathbf{V}-\mathbf{U}),
$$

where $a$ is the radius of the object and $d \ell=|\mathbf{V}-\mathbf{U}| d t$. Quantities $\mathbf{V}$ and $\mathbf{U}$ in equation (2) represent the velocities of the object and the particles of the medium. In writing equation (2), it has been assumed that, while sweeping up smaller particles, the density of the object remains unchanged and it stays perfectly spherical. It is important to emphasize that in this equation, the mass of the object, $m$, and its radius, $a$, are functions of time, and are related as

$$
\frac{d m}{d t}=\pi \rho_{\text {dust }}(r, z) a^{2} \frac{d \ell}{d t}
$$

Equation (3) immediately implies

$$
\frac{d a}{d t}=\frac{1}{4}\left(\frac{\rho_{\mathrm{dust}}}{\rho}\right) \frac{d \ell}{d t}
$$

where $\rho$ is the density of the object.

For small particles such as micron-sized and submicron-sized objects, one can replace $|\mathbf{V}-\mathbf{U}|$ with $\mathbf{V}_{\text {rel }}$, the relative velocity of the object with respect to the gas. The radial, vertical and tangential components of this velocity are, respectively, given by $\dot{r}, \dot{z}$, and $r\left(\dot{\varphi}-\omega_{g}\right)$, where the motions of gas molecules along the $z$-axis have been neglected. Because the gas is at hydrostatic equilibrium, its angular velocity, $\omega_{g}$, is slightly different from its Keplerian value and is given by

$$
\omega_{g}^{2}=\frac{G M}{\left(r^{2}+z^{2}\right)^{3 / 2}}+\frac{1}{r \rho_{g}(r, z)} \frac{\partial \mathscr{P}_{g}(r, z)}{\partial r} .
$$


In this equation, $\mathscr{P}_{g}(r, z)=K_{B} T \rho_{g}(r, z) / m_{H}$ is the pressure of the gas.

An object in the model nebula considered here is subject to the gravitational attraction of the central star and the drag force of the gas. The equation of motion of an object in this nebula is, therefore, given by

$$
m \ddot{\mathbf{R}}=-\frac{G M m}{\left(r^{2}+z^{2}\right)^{3 / 2}} \mathbf{R}-\pi \rho_{\text {dust }}(r, z) a^{2} \frac{d \ell}{d t} \mathbf{V}_{\text {rel }}-\mathbf{F}_{\text {drag }},
$$

where $\mathbf{R}(r, z)$ is the position vector of the particle and

$$
\mathbf{F}_{\text {drag }}=\frac{2 a^{2}}{3(a+\lambda)}\left(\frac{2 \pi K_{B} T}{m_{H}}\right)^{1 / 2}\left[\lambda \rho_{g}(r, z)+\frac{3 m_{H}}{2 \sigma}\right] \mathbf{V}_{\text {rel }}
$$

represents the drag force of the gas. In equation (7), $\sigma$ is the collisional cross section between two hydrogen molecules and $\lambda$ is their mean free path.

\section{NUMERICAL RESULTS}

The equation of motion of a particle and the growth equation (4) were integrated, numerically, for objects with initial radii ranging from 1 to 100 microns. The mass of the central star was chosen to be one solar mass, $\beta=1, r_{m}=1 \mathrm{AU}$, and $\rho_{0}=10^{-9} \mathrm{~g} \mathrm{~cm}^{-3}$. The collisional cross section of hydrogen molecules, $\sigma$, was taken to be $2 \times 10^{-15} \mathrm{~cm}^{-2}$, and their mean free paths $\lambda(\mathrm{cm})=4 \times 10^{-9} / \rho_{g}(r, z)\left(\mathrm{gcm}^{-3}\right)$. At the beginning of integrations, an object was placed at $(r, r / 10)$, with a Keplerian circular radial velocity and with no motion along the $z$-axis. Figure 1 shows the growth of two one micronsized objects, one migrating radially inward from $(2,0.2) \mathrm{AU}$, and one migrating radially outward from $(0.25,0.025)$ AU to $(1,0) \mathrm{AU}$, the location of the maximum gas density on the midplane. For the comparison, the radial and vertical motions of these objects without mass-growth have also been plotted. As shown here, by sweeping up the smaller particles of the background, these objects grow to a few centimeters in size and approach the midplane in a time much shorter than the time of similar migration without the massgrowth.

Equations (4) and (6) were also integrated for different values of the object's density and gas temperature. As expected, the rate of growth of the object increased with an increase in the temperature of the gas (Fig. 3). This can be attributed to larger value of the pressure gradients at higher temperatures [1]. Increasing the density of the object while keeping the temperature of the gas constant had, however, an opposite effect. Objects with higher densities tend to grow in size over longer periods of time. The inverse proportionality of the rate of the growth of the object to its density can also be seen from equation (4).

\section{ACKNOWLEDGMENTS}

This work is partially supported by the NASA Origins of the Solar System Program under grant NAG5-11569, and also the NASA Astrobiology Institute under Cooperative 

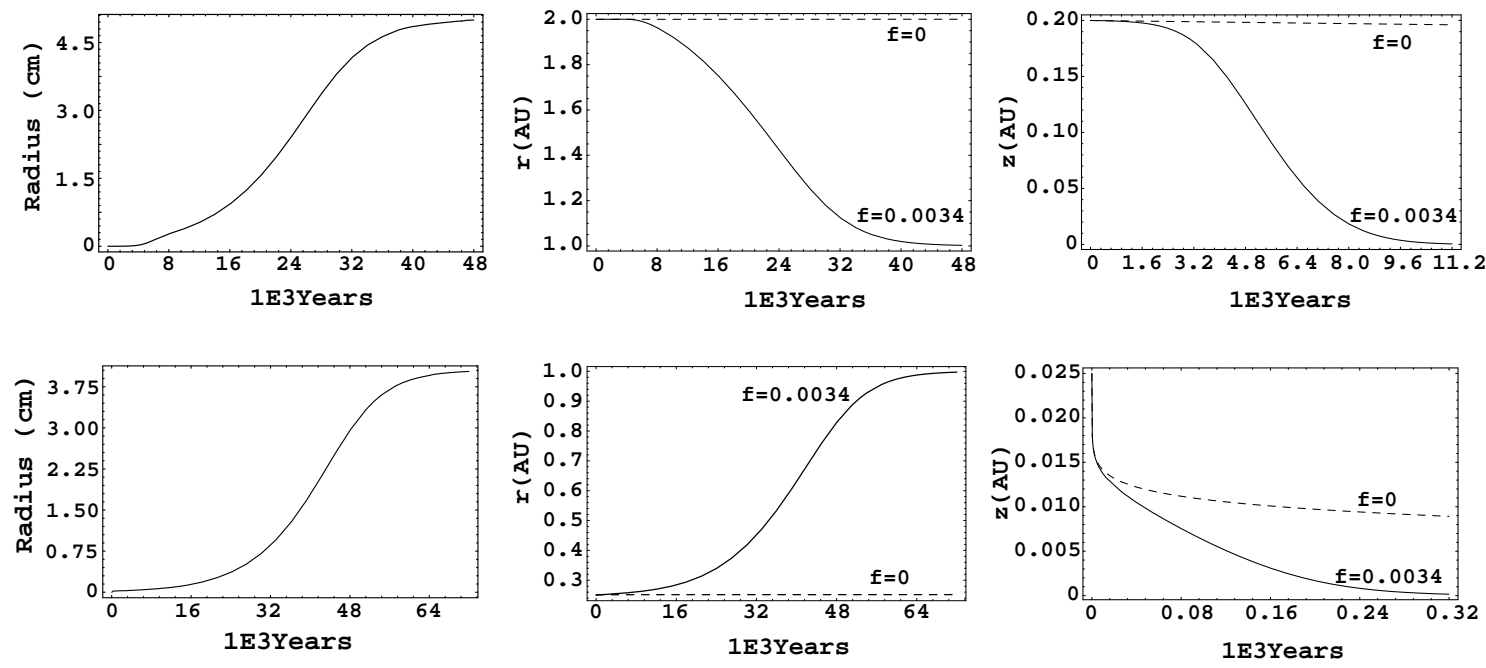

FIGURE 1. Graphs of the radius (left), radial migration (middle), and vertical descent (right) of a 1 micron object with a density of $2 \mathrm{~g} / \mathrm{cc}$. The gas temperature is $300 \mathrm{~K}$. The dashed line indicates migration without mass-growth. The middle graphs show an inward radial migration from 2 AU (top), and an outward radial migration from $0.25 \mathrm{AU}$ (bottom) to the location of the maximum gas density on the midplane, $(1,0)$ AU. The initial value of $z$ was taken to be $r / 10$.
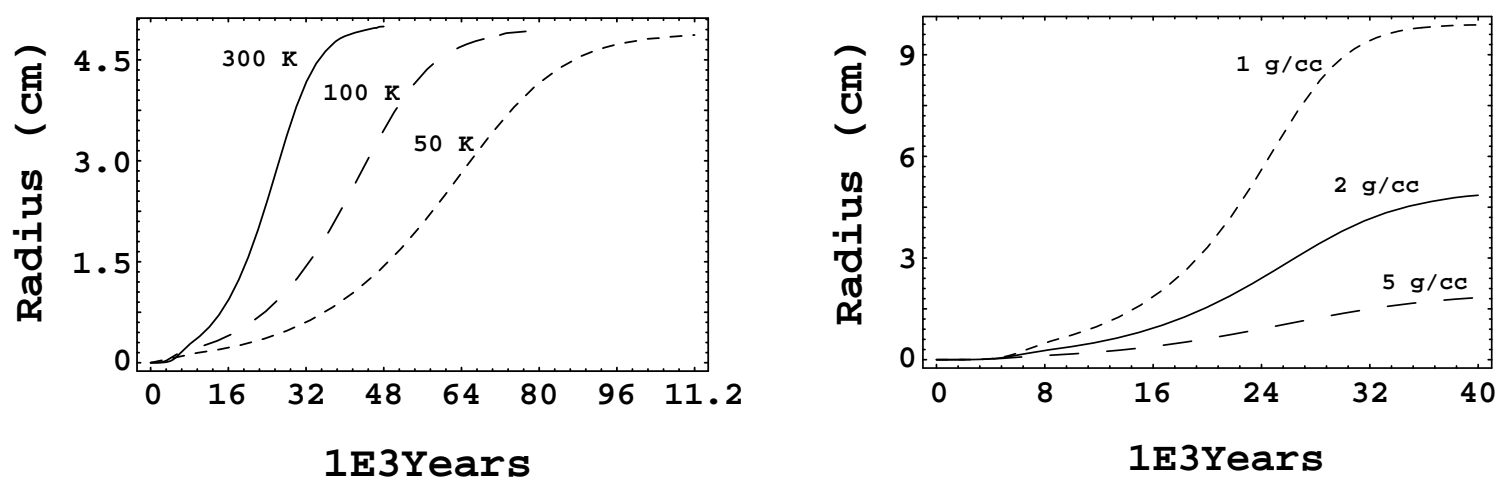

FIGURE 2. The growth of a 1 micron object with a density of $2 \mathrm{~g} / \mathrm{cc}$ for different values of the gas temperature is shown on the left. The right graph depicts the growth of similar particle with different densities in an isothermal gas with a temperature of $300 \mathrm{~K}$. The particle was initially at $(2,0.2) \mathrm{AU}$.

Agreement NCC2-1056.

\section{REFERENCES}

1. Haghighipour, H., and Boss, A. P., Astrophys. J., 583, 996-1003

2. Haghighipour, H., and Boss, A. P., Astrophys. J., 598, 1301-1311

On the Growth of Dust Particles in a Non-Uniform Solar Nebula

December 12, 2018 